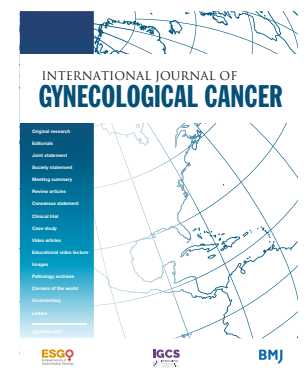

${ }^{1}$ Obstetrics and Gynecology, University of Toronto, Toronto, Ontario, Canada

${ }^{2}$ Obstetrics and Gynecology, Juravinski Cancer Centre, Hamilton, Ontario, Canada ${ }^{3}$ Gynecologic Oncology, Princess Margaret Cancer Center, University Health Network, Toronto, Ontario, Canada

Correspondence to Dr Taymaa May, Gynecologic Oncology, Princess Margaret Cancer Center, University Health Network, 610 University Avenue. OPG Wing, 6-811. Toronto, ON M5G 2M9, Canada; taymaa.may@uhn.ca

Accepted 12 April 2021 Published Online First 22 April 2021

\section{Assessment of anastomotic perfusion using indocyanine green fluorescence angiography following bowel resection for gynecologic malignancies: an instructional surgical video}

Elisabeth Spenard (D , ${ }^{1}$ Julie My Van Nguyen, ${ }^{2}$ Taymaa May ${ }^{1,3}$
Bowel resection is a procedure that is often conducted in the surgical management of patients with gynecologic malignancies. ${ }^{12}$ Real-time intraoperative assessment of anastomotic perfusion with indocyanine green fluorescence angiography (ICG$F A$ ) is a recent technique that has been described in the general surgery and gynecologic oncology literature. ${ }^{3}{ }^{4}$ Fluorescence angiography has been shown to be an effective intraoperative tool to assess perfusion of large and small bowel anastomoses. The objective of this video is to describe a step-by-step surgical approach to conducting
ICG-FA perfusion assessment for side-to-side and end-to-end circular anastomoses.

The video was built using surgical recordings of consented gynecologic oncology patients at a tertiary cancer center in Toronto, Canada between November 1, 2017 and September 1, 2020.

The five steps of this surgical technique are: (1) perform bowel anastomosis, (2) inspect and assess the anastomosis for mechanical integrity, (3) prepare near-infrared instrumentation and operative room setting, (4) administer $3 \mathrm{~mL}$ intravenous ICG (2.5 $\mathrm{mg} / \mathrm{mL}$ ) followed by $10 \mathrm{~mL}$ normal saline, and (5)

\section{INTRENATONAL OURRAL OF \\ GYNECOLOGICAL CANCER

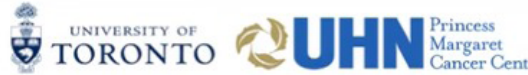

Assessment of Anastomotic Perfusion Using Indocyanine Green Fluorescence Angiography Following Bowel Resection for Gynecologic Malignancies: An Instructional Surgical Video Elisabeth Spénard, Julie M.V. Nguyen, Taymaa May*

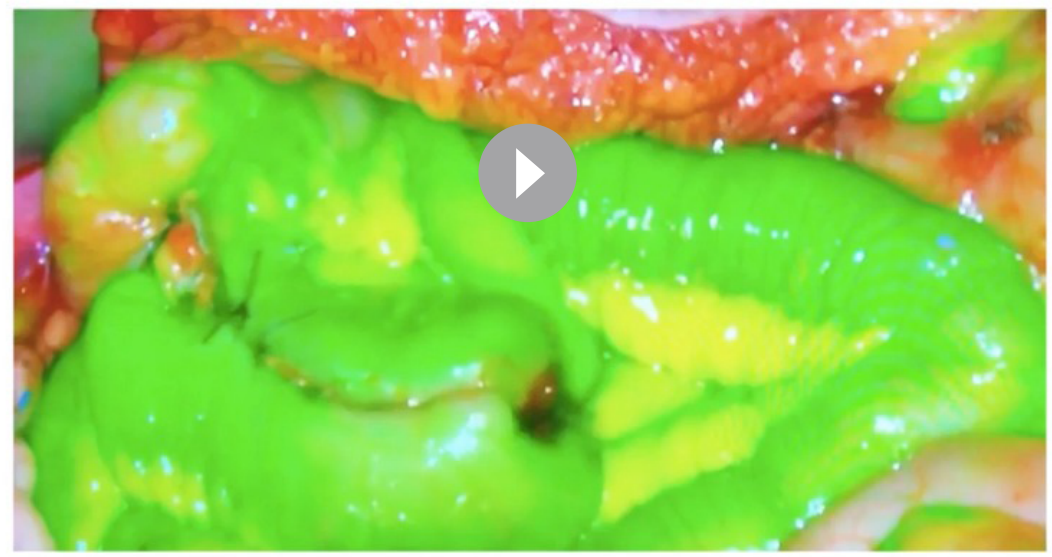
and permissions. Published by BMJ.

\begin{tabular}{l}
\hline To cite: Spenard E, \\
Nguyen JMV, May T. Int J \\
Gynecol Cancer 2021;31:932- \\
933. \\
\hline
\end{tabular}

\section{@IJGConline}

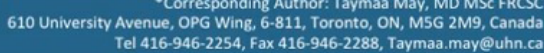
Copyrieht 02020 BMu Publishing Group Ltd, International Gynecologic Cancer Society, \& European Society of Gynaecological Oncology. All rights reserved.

Video 1 This surgical video describes a stepwise approach to the integration of indocyanine green fluorescence angiography (ICG-FA) into surgical practice following bowel resection which allows for standardized, reproducible, and objective assessment of anastomotic perfusion. 


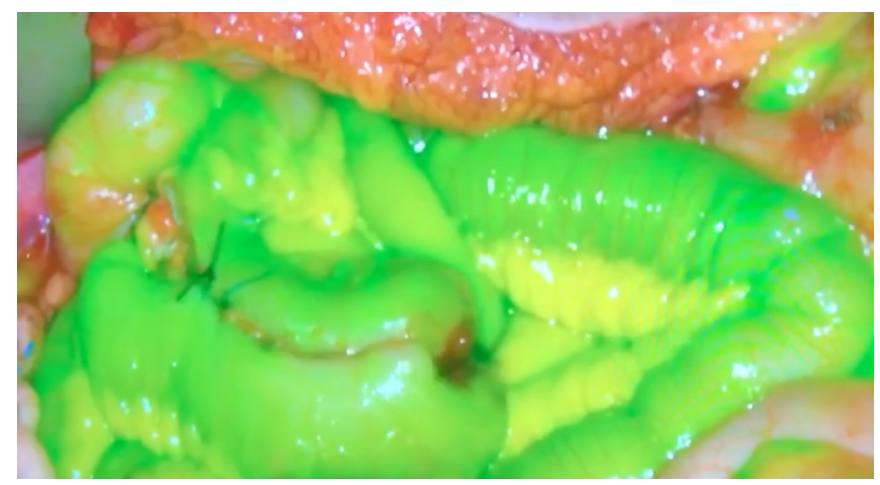

Figure 1 Adequate perfusion of the proximal and distal limbs of a side-to-side anastomosis is visualized using the portable hand-held near infrared imaging technology after administration of indocyanine green fluorescence angiography (ICG-FA).

observe and interpret anastomotic perfusion using near-infrared technology. For side-to-side anastomoses, perfusion assessment using ICG-FA was performed using a portable, hand-held, near-infrared imaging system transabdominally. For end-to-end circular anastomoses, an endoscopic near-infrared imaging system was used transanally through the transparent disposable shaft of a rigid sigmoidoscope, allowing assessment of perfusion of the colonic and rectal mucosa. An air leak test was also performed using the rigid sigmoidoscope. In both techniques, normal perfusion assessment with ICG-FA was defined as rapid ICG uptake of less than 1 min without segmental defects. Slower uptake over a minute, as assessed subjectively by the surgeon, or segmental perfusion defect were considered abnormal. Examples of normal and abnormal perfusion are shown in the video.

Objective assessment of anastomotic perfusion is of paramount importance in patients undergoing bowel resection and may decrease perioperative morbidity. Near-infrared perfusion assessment using ICG-FA is a promising tool that can be used with other risk-assessment strategies to guide operative decisionmaking in gynecologic oncology. This surgical video describes a clear stepwise approach to the integration of this technique into surgical practice which allows for standardized, reproducible, and objective assessment of anastomotic perfusion.

Contributors ES was responsible for conceptualization; methodology; data curation; video - montage, review, and editing; narration; writing - original draft; and writing - review and editing. JMVN was responsible for conceptualization; video - review and editing; and writing - review and editing. TM was responsible for conceptualization; methodology; data curation; video - image and video generation, copyright, review and editing; writing - review and editing; and supervision.

Funding The authors have not declared a specific grant for this research from any funding agency in the public, commercial or not-for-profit sectors.

Competing interests None declared.

Patient consent for publication Not required.

Provenance and peer review Not commissioned; externally peer reviewed.

Data availability statement All data relevant to the study are included in the article.

\section{ORCID iD}

Elisabeth Spenard http://orcid.org/0000-0001-6241-547X

\section{REFERENCES}

1 Vergote I, Tropé CG, Amant F, et al. Neoadjuvant chemotherapy or primary surgery in stage IIIC or IV ovarian cancer. N Engl J Med 2010;363:943-53.

2 Bakkum-Gamez JN, Langstraat CL, Martin JR, et al. Incidence of and risk factors for postoperative ileus in women undergoing primary staging and debulking for epithelial ovarian carcinoma. Gynecol Oncol 2012;125:614-20.

3 Blanco-Colino R, Espin-Basany E, RaEE-B B-C. Intraoperative use of ICG fluorescence imaging to reduce the risk of anastomotic leakage in colorectal surgery: a systematic review and meta-analysis. Tech Coloproctol 2018;22:15-23.

4 Nguyen JMV, Hogen L, Laframboise S, et al. The use of indocyanine green fluorescence angiography to assess anastomotic perfusion following bowel resection in surgery for gynecologic malignancies - a report of 100 consecutive anastomoses. Gynecol Oncol 2020;158:402-6. 